\title{
On the Taylor functional calculus
}

\author{
V. Müller \\ Mathematical Institute, Czech Academy of Sciences, Zitna 25, 11567 Prague 1, Czech Republic \\ E-mail: muller@math.cas.cz
}

\begin{abstract}
We give a Martinelli-Vasilescu type formula for the Taylor functional calculus and a simple proof of its basic properties.
\end{abstract}

Keywords and phrases: Taylor's functional calculus.

Let $A=\left(A_{1}, \ldots, A_{n}\right)$ be an $n$-tuple of mutually commuting operators acting on a Banach space $X$. The existence of the Taylor functional calculus [18], [19], for simpler versions see [10], [8], [3], [4], [5] and [15], is one of the most important results of spectral theory. However, the formula defining $f(A)$ for a function $f$ analytic on a neighbourhood of the Taylor spectrum has some drawbacks. The operator $f(A)$ is defined locally, the formula gives only $f(A) x$ for each $x \in X$. Therefore it is not easy to see that $f(A)$ is bounded. Moreover, the formula is rather inexplicit and it is quite difficult to prove even the basic properties of the calculus.

The situation is better for Hilbert space operators. In [20] and [21], Vasilescu gave an explicit Martinelli-type formula defining $f(A)$ which is much easier to handle.

The ideas of Vasilescu were used in [9] to prove a similar formula for Banach space operators. The method works, however, only for functions analytic on a neighbourhood of the split-spectrum which is in general bigger than the Taylor spectrum. The main tool is the existence of generalized inverses for operators that appear in the Koszul complex. For similar ideas see also [1].

In this paper we obtain a similar formula for the general Taylor functional calculus. The main innovation is the use of non-linear (but continuous) general inverses. In this way we obtain a formula that defines $f(A)$ globally, and so the continuity of $f(A)$ and the continuity of the functional calculus become clear. The formula is more explicit, and so it is possible to avoid some technical difficulties in the proof of the basic properties of the calculus. The cohomogical methods are avoided and the proofs are based only on the Stokes and the Bartle-Graves theorems.

The author wishes to thank to Professor F.-H. Vasilescu for numerous consultations concerning details of the calculus.

All Banach spaces in this paper are complex. Denote by $\mathcal{B}(X)$ the algebra of all bounded linear operators on a Banach space $X$.

Definition 1. Let $X, Y$ be Banach spaces. Denote by $\mathcal{H}(X, Y)$ the set of all continuous mappings $f: X \rightarrow Y$ that are homogeneous (i.e., $f(\alpha x)=\alpha f(x)$ for all $\alpha \in \mathbb{C}$ and $x \in X)$.

The research was supported by the grant no. 201/00/0208 of the Czech Academy of Sciences.

2000 Mathematics Subject Classification 47A60, 47A13. 
If $f \in \mathcal{H}(X, Y)$ then $\sup \{\|f(x)\|: x \in X,\|x\|=1\}<\infty$. Clearly $\mathcal{H}(X, Y)$ with this norm is a Banach space. Write for short $\mathcal{H}(X)$ instead of $\mathcal{H}(X, X)$. Clearly $\mathcal{B}(X) \subset \mathcal{H}(X)$.

Theorem 2. (Bartle-Graves, see [2], Proposition 5.9) Let $M$ be a closed subspace of a Banach space $X$ and let $\varepsilon>0$. Then there exists $h \in \mathcal{H}(X / M, X)$ such that $\|h\|<1+\varepsilon$ and $h(x+M) \in x+M$ for each class $x+M \in X / M$.

Lemma 3. Let $X, Y$ be Banach spaces and let $T: X \rightarrow Y$ be a bounded linear operator with closed range. Let $f \in \mathcal{H}(Y)$ satisfy $f(Y) \subset \operatorname{Im} T$. Then there exists $g \in \mathcal{H}(Y, X)$ such that $f=T g$.

Proof. Let $h: X / \operatorname{Ker} T \rightarrow X$ be the selection given by the Bartle-Graves theorem. Let $T_{0}: X / \operatorname{Ker} T \rightarrow \operatorname{Im} T$ be the operator induced by $T$. Set $g=h T_{0}^{-1} f$. For $y \in Y$ we have $T g y=T h T_{0}^{-1} f y=f y$, and so $T g=f$.

Q.E.D.

Proposition 4. Let $X_{0}, \ldots, X_{n}$ be Banach spaces, let $\delta_{j}: X_{j} \rightarrow X_{j+1} \quad(j=0, \ldots, n-$ 1) be bounded linear operators and suppose that the sequence

$$
0 \longrightarrow X_{0} \stackrel{\delta_{0}}{\longrightarrow} X_{1} \stackrel{\delta_{1}}{\longrightarrow} \cdots \stackrel{\delta_{n-1}}{\longrightarrow} X_{n} \longrightarrow 0
$$

is exact. Let $g_{j} \in \mathcal{H}\left(X_{j}\right) \quad(j=0, \ldots, n)$. The following statements are equivalent:

(i) $\delta_{j} g_{j}=g_{j+1} \delta_{j} \quad(j=0, \ldots, n-1)$;

(ii) there exist mappings $V_{j} \in \mathcal{H}\left(X_{j+1}, X_{j}\right) \quad(j=0, \ldots, n-1)$ such that

$$
\begin{aligned}
& V_{0} \delta_{0}=g_{0}, \\
& V_{j} \delta_{j}+\delta_{j-1} V_{j-1}=g_{j} \quad(j=1, \ldots, n-1), \\
& \delta_{n-1} V_{n-1}=g_{n} .
\end{aligned}
$$

Proof. (ii) $\Rightarrow\left(\right.$ i): Suppose that the mappings $V_{j}$ satisfy (ii). We have

$$
\delta_{j} g_{j}=\delta_{j}\left(V_{j} \delta_{j}+\delta_{j-1} V_{j-1}\right)=\delta_{j} V_{j} \delta_{j}
$$

and

$$
g_{j+1} \delta_{j}=\left(V_{j+1} \delta_{j+1}+\delta_{j} V_{j}\right) \delta_{j}=\delta_{j} V_{j} \delta_{j}
$$

(note that the same relations are true also for $j=0$ and $j=n-1$ ). Thus $\delta_{j} g_{j}=g_{j+1} \delta_{j}$ for all $j$.

(i) $\Rightarrow$ (ii): Since $\delta_{n-1}$ is onto, there exists $V_{n-1}$ such that $\delta_{n-1} V_{n-1}=g_{n}$.

We construct mappings $V_{j}$ inductively. Suppose that $1 \leq j \leq n-1$ and that $V_{j} \in \mathcal{H}\left(X_{j+1}, X_{j}\right)$ satisfies $V_{j+1} \delta_{j+1}+\delta_{j} V_{j}=g_{j+1}$ (for $j=n-1$ set formally $V_{n}=0$ and $\left.\delta_{n}=0\right)$. We have

$$
\delta_{j}\left(g_{j}-V_{j} \delta_{j}\right)=g_{j+1} \delta_{j}-\delta_{j} V_{j} \delta_{j}=g_{j+1} \delta_{j}-\left(g_{j+1}-V_{j+1} \delta_{j+1}\right) \delta_{j}=0 .
$$

Thus $\left(g_{j}-V_{j} \delta_{j}\right)\left(X_{j}\right) \subset \operatorname{Ker} \delta_{j}=\operatorname{Im} \delta_{j-1}$ and there exists $V_{j-1} \in \mathcal{H}\left(X_{j}, X_{j-1}\right)$ such that $\delta_{j-1} V_{j-1}=g_{j}-V_{j} \delta_{j}$. Thus $V_{j} \delta_{j}+\delta_{j-1} V_{j-1}=g_{j}$. 
At the end, suppose that $V_{0} \in \mathcal{H}\left(X_{1}, X_{0}\right)$ satisfies $g_{1}=V_{1} \delta_{1}+\delta_{0} V_{0}$. Then $\delta_{0} V_{0} \delta_{0}=$ $\left(g_{1}-V_{1} \delta_{1}\right) \delta_{0}=g_{1} \delta_{0}=\delta_{0} g_{0}$. Since $\delta_{0}$ is one-to-one, we have $V_{0} \delta_{0}=g_{0}$. This finishes the proof.

Q.E.D.

We recall now the basic notations of Taylor [18].

Denote by $\Lambda[s]$ the complex exterior algebra generated by the indeterminates $s=$ $\left(s_{1}, \ldots, s_{n}\right)$. Then

$$
\Lambda[s]=\bigoplus_{p=0}^{n} \Lambda^{p}[s]
$$

where $\Lambda^{p}[s]$ is the set of all elements of degree $p$ in $\Lambda[s]$. Thus the elements of $\Lambda^{p}[s]$ are of form

$$
\sum_{1 \leq i_{1}<\cdots<i_{p} \leq n} \alpha_{i_{1}, \ldots, i_{p}} s_{i_{1}} \wedge \cdots \wedge s_{i_{p}}
$$

where $\alpha_{i_{1}, \ldots, i_{p}}$ are complex numbers. The multiplication operation $\wedge$ is anticommutative, $s_{i} \wedge s_{j}=-s_{j} \wedge s_{i}$ for all $i, j$. In particular $s_{i} \wedge s_{i}=0$. Clearly $\operatorname{dim} \Lambda^{p}[s]=\left(\begin{array}{l}n \\ p\end{array}\right)$ and $\operatorname{dim} \Lambda[s]=2^{n}$.

Let $X$ be a Banach space. Then we write $\Lambda[s, X]=X \otimes \Lambda[s]$ and $\Lambda^{p}[s, X]=$ $X \otimes \Lambda^{p}[s]$. Thus the elements of $\Lambda^{p}[s, X]$ are of form

$$
\sum_{1 \leq i_{1}<\cdots<i_{p} \leq n} x_{i_{1}, \ldots, i_{p}} s_{i_{1}} \wedge \cdots \wedge s_{i_{p}}
$$

where $x_{i_{1}, \ldots, i_{p}} \in X$ (the symbol $\otimes$ is omitted in order to simplify the notation).

Let $A=\left(A_{1}, \ldots, A_{n}\right)$ be an $n$-tuple of mutually commuting operators in $X$. Define operator $\delta_{A}: \Lambda[s, X] \rightarrow \Lambda[s, X]$ by

$$
\delta_{A}\left(x s_{i_{1}} \wedge \cdots \wedge s_{i_{p}}\right)=\sum_{j=1}^{n}\left(A_{j} x\right) s_{j} \wedge s_{i_{1}} \wedge \cdots \wedge s_{i_{p}} .
$$

Write $\delta_{A}^{p}=\delta_{A} \mid \Lambda^{p}[s, X]$. The Koszul complex $\mathcal{K}(A)$ is the sequence

$$
0 \longrightarrow \Lambda^{0}[s, X] \stackrel{\delta_{A}^{0}}{\longrightarrow} \Lambda^{1}[s, X] \stackrel{\delta_{A}^{1}}{\longrightarrow} \cdots \stackrel{\delta_{A}^{n-1}}{\longrightarrow} \Lambda^{n}[s, X] \longrightarrow 0 .
$$

Then $\left(\delta_{A}\right)^{2}=0$, i.e., $\delta_{A}^{p} \delta_{A}^{p-1}=0$ for all $p$. It is convenient to set formally $\Lambda^{-1}[s, X]=\Lambda^{n+1}[s, X]=0$; similarly let $\delta_{A}^{-1}$ and $\delta_{A}^{n}$ be the zero operators.

We say that the $n$-tuple $A=\left(A_{1}, \ldots, A_{n}\right)$ is Taylor-regular if the Koszul complex $\mathcal{K}(A)$ is exact (i.e., $\left.\operatorname{Im} \delta_{A}=\operatorname{Ker} \delta_{A}\right)$. The Taylor spectrum $\sigma_{T}(A)$ is the set of all $n$-tuples $\lambda=\left(\lambda_{1}, \ldots, \lambda_{n}\right) \in \mathbb{C}^{n}$ such that $A-\lambda=\left(A_{1}-\lambda_{1}, \ldots, A_{n}-\lambda_{n}\right)$ is not Taylor-regular. It is well-known that $\sigma_{T}(A)$ is a nonempty compact subset of $\mathbb{C}^{n}$. Further, the Taylor spectrum satisfies the projection property, see [18], [16].

Let $A=\left(A_{1}, \ldots, A_{n}\right)$ be a Taylor-regular $n$-tuple of operators. By Proposition 4 , there are "generalized inverses" $V_{j} \in \mathcal{H}\left(\Lambda^{j+1}[s, X], \Lambda^{j}[s, X]\right)$ such that $\delta_{A}^{j-1} V_{j-1}+$ $V_{j} \delta_{A}^{j}=I_{\Lambda^{j}[s, X]}$. In a simpler form, we have $\delta_{A} V+V \delta_{A}=I_{\Lambda[s, X]}$ where $V \in \mathcal{H}(\Lambda[s, X])$ is defined by $V\left(\oplus_{j=0}^{n} \psi_{j}\right)=\oplus_{j=1}^{n} V_{j-1} \psi_{j} \quad\left(\psi_{j} \in \Lambda^{j}[s, X]\right)$.

Our first goal is to show that it is possible to find such generalized inverses depending smoothly on $z \in \mathbb{C}^{n} \backslash \sigma_{T}(A)$. 
Proposition 5. Let $A=\left(A_{1}, \ldots, A_{n}\right)$ be an $n$-tuple of mutually commuting operators on a Banach space $X$. Let $G=\mathbb{C}^{n} \backslash \sigma_{T}(A)$. Then there exists a $C^{\infty}$ - function $V: G \rightarrow \mathcal{H}(\Lambda[s, X])$ such that $\delta_{A-z} V(z)+V(z) \delta_{A-z}=I_{\Lambda[s, X]}$, and

$$
V(z) \Lambda^{p}[s, X] \subset \Lambda^{p-1}[s, X] \quad(z \in G, p=0, \ldots, n) .
$$

Proof. Consider Banach spaces

$$
\begin{aligned}
M_{1} & =\bigoplus_{j=0}^{n-1} \mathcal{H}\left(\Lambda^{j+1}[s, X], \Lambda^{j}[s, X]\right), \\
M_{2} & =\bigoplus_{j=0}^{n} \mathcal{H}\left(\Lambda^{j}[s, X]\right) \text { and } \\
M_{3} & =\bigoplus_{j=0}^{n-1} \mathcal{H}\left(\Lambda^{j}[s, X], \Lambda^{j+1}[s, X]\right) .
\end{aligned}
$$

For $z \in G$ define mappings $\Phi(z): M_{1} \rightarrow M_{2}$ and $\Psi(z): M_{2} \rightarrow M_{3}$ by

$$
\Phi(z)\left(\bigoplus_{j=0}^{n-1} V_{j}\right)=V_{0} \delta_{A-z}^{0} \oplus \bigoplus_{j=1}^{n-1}\left(V_{j} \delta_{A-z}^{j}+\delta_{A-z}^{j-1} V_{j-1}\right) \oplus \delta_{A-z}^{n-1} V_{n-1}
$$

and

$$
\Psi(z)\left(\bigoplus_{j=0}^{n} g_{j}\right)=\bigoplus_{j=0}^{n-1}\left(\delta_{A-z}^{j} g_{j}-g_{j+1} \delta_{A-z}^{j}\right)
$$

Clearly $\Phi(z)$ and $\Psi(z)$ are bounded linear operators depending analytically on $z \in G$ and, by Proposition $4, \operatorname{Im} \Phi(z)=\operatorname{Ker} \Psi(z)$. Further $I_{\Lambda[s, X]}=\oplus I_{\Lambda^{i}[s, X]} \in \operatorname{Im} \Phi(z)$ for all $z \in G$.

Let $\lambda \in G$. By [18], Lemma 2.2, cf. also [17], there is a neighbourhood $U_{\lambda}$ of $\lambda$ and an analytic function $V_{\lambda}: U_{\lambda} \rightarrow M_{1}$ such that $\Phi(z) V_{\lambda}(z)=I \quad\left(z \in U_{\lambda}\right)$.

Let $\left\{\varphi_{i}\right\}_{i=1}^{\infty}$ be a $C^{\infty}$-partition of unity subordinated to the cover $\left\{U_{\lambda}, \lambda \in G\right\}$ of $G$, i.e. $\varphi_{i}$ 's are $C^{\infty}$-functions, $0 \leq \varphi_{i} \leq 1$, supp $\varphi_{i} \subset U_{\lambda_{i}}$ for some $\lambda_{i} \in G$, for each $\lambda \in G$ there exists a neighbourhood $U$ of $\lambda$ such that all but finitely many of $\varphi_{i}$ 's are 0 on $U$ and $\sum_{i=1}^{\infty} \varphi_{i}(z)=1$ for each $z \in G$.

For $z \in G$ set $V(z)=\sum_{i=1}^{\infty} \varphi_{i}(z) V_{\lambda_{i}}(z)$. Clearly $V$ is a $C^{\infty}$-function satisfying $V(z) \Lambda^{p}[s, X] \subset \Lambda^{p-1}[s, X]$ and $\Phi(z) V(z)=I$ for all $z \in G$.

Q.E.D.

Remark 6. (i) Function $\Phi$ is regular in $G$ (i.e., $\operatorname{Im} \Phi(z)$ changes continuously). The existence of a $C^{\infty}$-function $V$ satisfying $\Phi(z) V(z)=I$ follows also directly from a deep result of Mantlik [11]. The present argument, however, is more elementary.

(ii) It is possible to require also that $V(z)^{2}=0$ and $V(z) \delta_{A-z} V(z)=V(z)$ for all $z \in G$. In particular, $V(z)$ is a generalized inverse of $\delta_{A-z}$.

Indeed, let $V: G \rightarrow \mathcal{H}(\Lambda[s, X])$ be the function constructed in Proposition 5, i.e., $\delta_{A-z} V(z)+V(z) \delta_{A-z}=I$ and $V(z) \Lambda^{p}[s, X] \subset \Lambda^{p-1}[s, X]$. 
Clearly $\delta_{A-z} V(z) \delta_{A-z}=\delta_{A-z}$. Set $V^{\prime}(z)=V(z) \delta_{A-z} V(z)$. Then

$$
\delta_{A-z} V^{\prime}(z) \delta_{A-z}=\delta_{A-z} V(z) \delta_{A-z} V(z) \delta_{A-z}=\delta_{A-z}
$$

and

$$
V^{\prime}(z) \delta_{A-z} V^{\prime}(z)=V(z) \delta_{A-z} V(z) \delta_{A-z} V(z) \delta_{A-z} V(z)=V(z) \delta_{A-z} V(z)=V^{\prime}(z) .
$$

Further

$$
\begin{aligned}
& \delta_{A-z} V^{\prime}(z)+V^{\prime}(z) \delta_{A-z}=\delta_{A-z} V(z) \delta_{A-z} V(z)+V(z) \delta_{A-z} V(z) \delta_{A-z} \\
& =\delta_{A-z} V(z)+V(z) \delta_{A-z}=I .
\end{aligned}
$$

Finally we have

$$
V^{\prime}(z)=\left(V^{\prime}(z) \delta_{A-z}+\delta_{A-z} V^{\prime}(z)\right) V^{\prime}(z)=V^{\prime}(z)+\delta_{A-z} V^{\prime}(z)^{2},
$$

and so $\delta_{A-z} V^{\prime}(z)^{2}=0$. Thus $V^{\prime}(z)^{2}=\left(V^{\prime}(z) \delta_{A-z}+\delta_{A-z} V^{\prime}(z)\right) V^{\prime}(z)^{2}=0$.

These additional properties of the generalized inverse $V$, however, are not essential for our purpose and we are not going to use them in the sequel.

In the following we fix a commuting $n$-tuple $A=\left(A_{1}, \ldots, A_{n}\right)$ of bounded linear operators on a Banach space $X$, the set $G=\mathbb{C}^{n} \backslash \sigma_{T}(A)$ and a $C^{\infty}$-function $V: G \rightarrow$ $\mathcal{H}(\Lambda[s, X])$ with the properties of Proposition 5 .

Consider the space $C^{\infty}(G, \Lambda[s, X])$. Clearly this space can be identified with the set $\Lambda\left[s, C^{\infty}(G, X)\right]$.

Function $V: G \rightarrow \mathcal{H}(\Lambda[s, X])$ induces naturally the operator (denoted by the same symbol) $V: C^{\infty}(G, \Lambda[s, X]) \rightarrow C^{\infty}(G, \Lambda[s, X])$ by

$$
(V y)(z)=V(z) y(z) \quad\left(z \in G, y \in C^{\infty}(G, \Lambda[s, X])\right) .
$$

Similarly we define operator $\delta_{A-z}$ (or $\delta$ for short if no ambiguity can arise) acting in $C^{\infty}(G, \Lambda[s, X])$ by

$$
(\delta y)(z)=\delta_{A-z} y(z) \quad\left(z \in G, y \in C^{\infty}(G, \Lambda[s, X])\right) .
$$

Clearly $\delta^{2}=0, V \delta+\delta V=I_{\Lambda\left[s, C^{\infty}(G, X)\right]}$ and both $V$ and $\delta$ are "graded", i.e.

$$
\begin{aligned}
& V \Lambda^{p}\left[s, C^{\infty}(G, X)\right] \subset \Lambda^{p-1}\left[s, C^{\infty}(G, X)\right] \quad \text { and } \\
& \delta \Lambda^{p}\left[s, C^{\infty}(G, X)\right] \subset \Lambda^{p+1}\left[s, C^{\infty}(G, X)\right] .
\end{aligned}
$$

Consider now another indeterminates $\mathrm{d} \bar{z}=\left(\mathrm{d} \bar{z}_{1}, \ldots, \mathrm{d} \bar{z}_{n}\right)$ and the space $\Lambda\left[s, \mathrm{~d} \bar{z}, C^{\infty}(G, X)\right]$. Define the linear operator

$$
\bar{\partial}: \Lambda\left[s, \mathrm{~d} \bar{z}, C^{\infty}(G, X)\right] \rightarrow \Lambda\left[s, \mathrm{~d} \bar{z}, C^{\infty}(G, X)\right]
$$

by

$$
\bar{\partial} f s_{i_{1}} \wedge \ldots \wedge s_{i_{p}} \wedge \mathrm{d} \bar{z}_{j_{1}} \wedge \ldots \wedge \mathrm{d} \bar{z}_{j_{q}}=\sum_{k=1}^{n} \frac{\partial f}{\partial \bar{z}_{k}} \mathrm{~d} \bar{z}_{k} \wedge s_{i_{1}} \wedge \ldots \wedge s_{i_{p}} \wedge \mathrm{d} \bar{z}_{j_{1}} \wedge \ldots \wedge \mathrm{d} \bar{z}_{j_{q}} .
$$


Clearly $\bar{\partial}^{2}=0$.

Operators $V$ and $\delta$ can be lifted from $\Lambda\left[s, C^{\infty}(G, X)\right]$ to $\Lambda\left[s, \mathrm{~d} \bar{z}, C^{\infty}(G, X)\right]$ by

$$
\begin{aligned}
V\left(\psi \wedge \mathrm{d} \bar{z}_{i_{1}} \wedge \ldots \mathrm{d} \bar{z}_{i_{p}}\right) & =(V \psi) \wedge \mathrm{d} \bar{z}_{i_{1}} \wedge \ldots \mathrm{d} \bar{z}_{i_{p}} \quad \text { and } \\
\delta\left(\psi \wedge \mathrm{d} \bar{z}_{i_{1}} \wedge \ldots \mathrm{d} \bar{z}_{i_{p}}\right) & =(\delta \psi) \wedge \mathrm{d} \bar{z}_{i_{1}} \wedge \ldots \mathrm{d} \bar{z}_{i_{p}}
\end{aligned}
$$

for all $\psi \in \Lambda\left[s, C^{\infty}(G, X)\right]$. Clearly the properties of $V$ and $\delta$ are preserved: $\delta^{2}=0$, $V \delta+\delta V=I$ and both $V$ and $\delta$ are graded. Note also that $\delta \bar{\partial}=-\bar{\partial} \delta$ and $(\bar{\partial}+\delta)^{2}=0$.

Let $W: \Lambda\left[s, \mathrm{~d} \bar{z}, C^{\infty}(G, X)\right] \rightarrow \Lambda\left[s, \mathrm{~d} \bar{z}, C^{\infty}(G, X)\right]$ be the mapping defined in the following way: if $\psi \in \Lambda\left[s, \mathrm{~d} \bar{z}, C^{\infty}(G, X)\right], \psi=\psi_{0}+\cdots+\psi_{n}$ where $\psi_{j}$ is the part of $\psi$ of degree $j$ in $\mathrm{d} \bar{z}$, then set $W \psi=\eta_{0}+\cdots+\eta_{n}$ where

$$
\begin{aligned}
\eta_{0} & =V \psi_{0}, \\
\eta_{1} & =V\left(\psi_{1}-\bar{\partial} \eta_{0}\right), \\
& \vdots \\
\eta_{n} & =V\left(\psi_{n}-\bar{\partial} \eta_{n-1}\right) .
\end{aligned}
$$

Note that $\eta_{j}$ is the part of $W \psi$ of degree $j$ in $\mathrm{d} \bar{z}$.

Lemma 7. Let $W: \Lambda\left[s, \mathrm{~d} \bar{z}, C^{\infty}(G, X)\right] \rightarrow \Lambda\left[s, \mathrm{~d} \bar{z}, C^{\infty}(G, X)\right]$ be the mapping defined above. Then:

(i) $\operatorname{supp} W \psi \subset \operatorname{supp} \psi$ for all $\psi$;

(ii) if $G^{\prime}$ is an open subset of $G$ and $\psi \in \Lambda\left[s, \mathrm{~d} \bar{z}, C^{\infty}(G, X)\right]$ satisfies $(\bar{\partial}+\delta) \psi=0$ on $G^{\prime}$, then $(\bar{\partial}+\delta) W \psi=\psi$ on $G^{\prime}$

(iii) $(\bar{\partial}+\delta) W(\bar{\partial}+\delta)=\bar{\partial}+\delta$.

Proof. (i) Clear.

(ii) Let $\psi=\psi_{0}+\cdots+\psi_{n}$ where $\psi_{j}$ is the part of $\psi$ of degree $j$ in $\mathrm{d} \bar{z}$. Condition $(\bar{\partial}+\delta) \psi=0$ on $G^{\prime}$ can be rewritten as

$$
\begin{aligned}
\delta \psi_{0} & =0 \\
\bar{\partial} \psi_{0}+\delta \psi_{1} & =0 \\
\vdots & \\
\bar{\partial} \psi_{n-1}+\delta \psi_{n} & =0
\end{aligned}
$$

(condition $\bar{\partial} \psi_{n}=0$ is satisfied automatically).

Let $W \psi=\eta_{0}+\cdots+\eta_{n}$ where $\eta_{j}$ are defined by (1). The required condition $(\bar{\partial}+\delta) W \psi=\psi$ then becomes

$$
\begin{aligned}
\delta \eta_{0} & =\psi_{0}, \\
\bar{\partial} \eta_{0}+\delta \eta_{1} & =\psi_{1}, \\
& \vdots \\
\bar{\partial} \eta_{n-1}+\delta \eta_{n} & =\psi_{n}
\end{aligned}
$$


on $G^{\prime}$ (again, $\bar{\partial} \eta_{n}=0$ automatically).

By (1) and (2), we have $\delta \eta_{0}=\delta V \psi_{0}=(\delta V+V \delta) \psi_{0}=\psi_{0}$ and $\bar{\partial} \eta_{0}+\delta \eta_{1}=$ $\bar{\partial} \eta_{0}+\delta V\left(\psi_{1}-\bar{\partial} \eta_{0}\right)=\bar{\partial} \eta_{0}+(I-V \delta)\left(\psi_{1}-\bar{\partial} \eta_{0}\right) \bar{\partial} \eta_{0}=\psi_{1}-V \delta\left(\psi_{1}-\bar{\partial} \eta_{0}\right)=\psi_{1}$ since $\delta\left(\psi_{1}-\bar{\partial} \eta_{0}\right)=\delta \psi_{1}+\bar{\partial} \delta \eta_{0}=\delta \psi_{1}+\bar{\partial} \psi_{0}=0$.

We prove (3) by induction. Suppose that $\bar{\partial} \eta_{j-1}+\delta \eta_{j}=\psi_{j}$ for some $j \geq 1$. Then $\delta\left(\psi_{j+1}-\bar{\partial} \eta_{j}\right)=\delta \psi_{j+1}+\bar{\partial} \delta \eta_{j}=\delta \psi_{j+1}+\bar{\partial} \psi_{j}=0$ by the induction assumption, and $\bar{\partial} \eta_{j}+\delta \eta_{j+1}=\bar{\partial} \eta_{j}+\delta V\left(\psi_{j+1}-\bar{\partial} \eta_{j}\right)=\bar{\partial} \eta_{j}+(I-V \delta)\left(\psi_{j+1}-\bar{\partial} \eta_{j}\right)=\psi_{j+1}$.

(iii) Since $(\bar{\partial}+\delta)^{2}=0$, the statement follows from (ii).

Q.E.D.

Remark 8. Without any change it is possible to prove the preceding theorem in a more general form. Let $z \mapsto A(z)$ be an analytic function defined on an open subset $G \subset \mathbb{C}^{n}$ such that the values $A(z)$ are Taylor regular $n$-tuples of operators on $X$ for all $z \in G$. Let $\psi \in \Lambda\left[s, \mathrm{~d} \bar{z}, C^{\infty}(G, X)\right]$ satisfy $\left(\bar{\partial}+\delta_{A(z)}\right) \psi=0$. Then there exists a form $\theta \in \Lambda\left[s, \mathrm{~d} \bar{z}, C^{\infty}(G, X)\right]$ with $\operatorname{supp} \theta \subset \operatorname{supp} \psi$ and $\psi=\left(\bar{\partial}+\delta_{A(z)}\right) \theta$.

We interpret the differential form

$$
(2 i)^{-n} \mathrm{~d} \bar{z}_{1} \wedge \cdots \wedge \mathrm{d} \bar{z}_{n} \wedge \mathrm{d} z_{1} \wedge \cdots \wedge \mathrm{d} z_{n}
$$

as the Lebesgue measure in $\mathbb{C}^{n}=\mathbb{R}^{2 n}$.

Let $P$ be the natural projection $P: \Lambda\left[s, \mathrm{~d} \bar{z}, C^{\infty}(G, X)\right] \rightarrow \Lambda\left[\mathrm{d} \bar{z}, C^{\infty}(G, X)\right]$ that annihilates all terms containing at least one of the indeterminates $s_{1}, \ldots, s_{n}$ and leaves invariant all the remaining terms.

The following simple lemma will be used frequently.

Proposition 9. Let $\eta \in \Lambda^{n}\left[s, d \bar{z}, C^{\infty}(G, X)\right]$ be a differential form with a compact support disjoint with $\sigma_{T}(A)$ such that $(\bar{\partial}+\delta) \eta=0$. Then

$$
\int_{\mathbb{C}^{n}} P \eta \wedge \mathrm{d} z=0
$$

where $\mathrm{d} z$ stands for $\mathrm{d} z_{1} \wedge \cdots \wedge \mathrm{d} z_{n}$.

Proof. We have

$$
P \eta=P(\bar{\partial}+\delta) W \eta=P \bar{\partial} W \eta=\bar{\partial} P W \eta
$$

where $P W \eta$ has a compact support. By the Stokes theorem, we have

$$
\int_{\mathbb{C}^{n}} P \eta \wedge \mathrm{d} z=\int_{\mathbb{C}^{n}} \bar{\partial} P W \eta \wedge \mathrm{d} z=0
$$

Q.E.D.

Let $U$ be a neighbourhood of $\sigma_{T}(A)$. It is possible to find a compact neighbourhood $\Delta$ of $\sigma_{T}(A)$ such that $\Delta \subset U$ and the boundary $\partial \Delta$ is a smooth surface. Let $f$ be a function analytic in $U$. Define operator $f(A)$ by

$$
f(A) x=\frac{-1}{(2 \pi i)^{n}} \int_{\partial \Delta} P W f(z) x s \wedge \mathrm{d} z \quad(x \in X),
$$


where $\mathrm{d} z$ stands for $\mathrm{d} z_{1} \wedge \cdots \wedge \mathrm{d} z_{n}$ and $s=s_{1} \wedge \cdots \wedge s_{n}$. By the Stokes formula,

$$
f(A) x=\frac{-1}{(2 \pi i)^{n}} \int_{\Delta} \bar{\partial} \varphi P W f(z) x s \wedge \mathrm{d} z
$$

where $\varphi$ is a $C^{\infty}$-function equal to 0 on a neighbourhood of $\sigma_{T}(A)$ and to 1 on $\mathbb{C}^{n} \backslash \Delta$ (consequently, $\varphi=1$ also on $\partial \Delta$ ).

On $\mathbb{C}^{n} \backslash \Delta$ we have

$$
\bar{\partial} \varphi P W f x s=P(\bar{\partial}+\delta) W f x s=P f x s=0 .
$$

Thus we can write

$$
f(A) x=\frac{-1}{(2 \pi i)^{n}} \int_{\mathbb{C}^{n}} \bar{\partial} \varphi P W f(z) x s \wedge \mathrm{d} z .
$$

It is clear from the Stokes theorem that the definition of $f(A) x$ does not depend on the choice of function $\varphi$ and, by (6), it is independent of $\Delta$.

We show that $f(A)$ does not depend on the choice of the generalized inverse $V$ which determines $W$.

Suppose that $W_{1}, W_{2}$ are two operators satisfying

$$
(\bar{\partial}+\delta) W_{i} f(z) x s=f(z) x s \quad(i=1,2) .
$$

For those $z$ where $\varphi \equiv 1$ we have

$$
(\bar{\partial}+\delta) \varphi\left(W_{1}-W_{2}\right) f(z) x s=0,
$$

and so the form $(\bar{\partial}+\delta) \varphi\left(W_{1}-W_{2}\right) f(z) x s$ satisfies the conditions of Proposition 9. Hence

$$
\begin{gathered}
0=\int_{\mathbb{C}^{n}} P(\bar{\partial}+\delta) \varphi\left(W_{1}-W_{2}\right) f(z) x s \wedge \mathrm{d} z=\int_{\mathbb{C}^{n}} P \bar{\partial} \varphi\left(W_{1}-W_{2}\right) f(z) x s \wedge \mathrm{d} z= \\
=\int_{\mathbb{C}^{n}} \bar{\partial} \varphi P W_{1} f(z) x s \wedge \mathrm{d} z-\int_{\mathbb{C}^{n}} \bar{\partial} \varphi P W_{2} f(z) x s \wedge \mathrm{d} z .
\end{gathered}
$$

It is possible to express the mapping $P W$ that appears in the definition of the functional calculus more explicitly. By the definition of $W$, we have

$$
P W x s=(-1)^{n-1} V(\bar{\partial} V)^{n-1} x s=(-1)^{n-1} V_{0} \bar{\partial} V_{1} \bar{\partial} \cdots \bar{\partial} V_{n-1} x s .
$$

Since $\Lambda[s, X]$ is a direct sum of $2^{n}$ copies of $X$, we can express $V(z): \Lambda[s, X] \rightarrow$ $\Lambda[s, X]$ in the matrix form whose entries are elements of $\mathcal{H}(X)$ depending smoothly on $z \in G$.

Clearly we can write $P W x s=\sum_{i=1}^{n} M^{(i)} x \mathrm{~d} \bar{z}_{1} \wedge \cdots \widehat{\mathrm{d} \bar{z}_{i}} \cdots \wedge \mathrm{d} \bar{z}_{n}$ for certain functions $M^{(i)} \in C^{\infty}(G, \mathcal{H}(X))$ where the hat denotes the omitted term.

Thus we can write formulas (5) and (6) also globally:

$$
\begin{aligned}
f(A) & =\frac{-1}{(2 \pi i)^{n}} \int_{\partial \Delta} P W f(z) I s \wedge \mathrm{d} z=\frac{-1}{(2 \pi i)^{n}} \int_{\mathbb{C}^{n}} \bar{\partial} \varphi P W f(z) I s \wedge \mathrm{d} z \\
& =\frac{(-1)^{n}}{(2 \pi i)^{n}} \int_{\mathbb{C}^{n}} \bar{\partial} \varphi V(\bar{\partial} V)^{n-1} f(z) I s \wedge \mathrm{d} z
\end{aligned}
$$

where $I=I_{X}$ is the identity operator on $X$. The coefficients of forms in (7) are $\mathcal{H}(X)$-valued $C^{\infty}$-functions. Therefore $f(A) \in \mathcal{H}(X)$. 
Lemma 10. $f(A)$ is a bounded linear operator.

Proof. Since $f(A) \in \mathcal{H}(X)$, it is sufficient to show only the additivity. Let $x, y \in X$. Let $\varphi$ be a $C^{\infty}$-function equal to 0 on a neighbourhood of $\sigma_{T}(A) \operatorname{such}$ that $\operatorname{supp}(1-\varphi)$ is compact. Then

$$
\begin{aligned}
& -(2 \pi i)^{n}(f(A)(x+y)-f(A) x-f(A) y) \\
& =\int_{\mathbb{C}^{n}} \bar{\partial} \varphi P W f \cdot(x+y) s \wedge \mathrm{d} z-\int_{\mathbb{C}^{n}} \bar{\partial} \varphi P W f x s \wedge \mathrm{d} z-\int_{\mathbb{C}^{n}} \bar{\partial} \varphi P W f y s \wedge \mathrm{d} z \\
& =\int_{\mathbb{C}^{n}} P \eta \wedge \mathrm{d} z
\end{aligned}
$$

where

$$
\eta=(\bar{\partial}+\delta) \varphi W f \cdot(x+y) s-(\bar{\partial}+\delta) \varphi W f x s-(\bar{\partial}+\delta) \varphi W f y s .
$$

Clearly $\eta$ has a compact support disjoint with $\sigma_{T}(A)$ and $(\bar{\partial}+\delta) \eta=0$. By Proposition 9, $\int P \eta \wedge \mathrm{d} z=0$ and $f(A)(x+y)=f(A) x+f(A) y$.

Q.E.D.

Proposition 11. For $n=1$ the functional calculus defined by (7) coincides with the classical functional calculus given by the Cauchy formula.

Proof. Let $A \in \mathcal{B}(X)$ and let $f$ be a function analytic on a neighbourhood of $\sigma(A)$. Then $W x s=V x s=(A-z)^{-1} x$. Thus, for a suitable contour $\Sigma$ surrounding $\sigma(A)$, we have

$$
f(A)=\frac{-1}{2 \pi i} \int_{\Sigma} P W f I s \wedge \mathrm{d} z=\frac{-1}{2 \pi i} \int_{\Sigma}(A-z)^{-1} f(z) I \mathrm{~d} z=\frac{1}{2 \pi i} \int_{\Sigma} f(z)(z-A)^{-1} \mathrm{~d} z
$$

which is the Cauchy formula.

Q.E.D.

Proposition 12. Let $f$ be a function analytic on a neighbourhood of $\sigma_{T}(A), 1 \leq j \leq n$ and $g(z)=z_{j} f(z)$. Then $g(A)=A_{j} f(A)$.

Proof. The statement is well-known for $n=1$. Suppose that $n \geq 2$. Then

$$
\begin{aligned}
-(2 \pi i)^{n}\left(A_{j} f(A)-g(A)\right) & =A_{j} \int_{\mathbb{C}^{n}} \bar{\partial} \varphi P W f I s \wedge \mathrm{d} z-\int_{\mathbb{C}^{n}} \bar{\partial} \varphi P W z_{j} f I s \wedge \mathrm{d} z \\
& =\int_{\mathbb{C}^{n}} \bar{\partial} \varphi f \cdot\left(A_{j}-z_{j}\right) P W I s \wedge \mathrm{d} z .
\end{aligned}
$$

For $F \subset\{1, \ldots, n\}, F=\left\{i_{1}, \ldots, i_{p}\right\}$ where $i_{1}<i_{2}<\cdots<i_{p}$ write $s_{F}=s_{i_{1}} \wedge \cdots \wedge s_{i_{p}}$. Express $W I s \in \Lambda^{n-1}\left[s, \mathrm{~d} \bar{z}, C^{\infty}(G, X)\right]$ as

$$
W I s=\sum_{F \subset\{1, \ldots, n\}} s_{F} \wedge \xi_{F}
$$

where $\xi_{F}$ contains no variable from $s_{1}, \ldots, s_{n}$. Since $\left(\bar{\partial}+\delta_{A-z}\right) W I s=I s$, for each $F \neq\{1, \ldots, n\}$ we have

$$
\bar{\partial} \xi_{F}+\sum_{k \in F}(-1)^{\operatorname{card}\left\{k^{\prime} \in F: k^{\prime}<k\right\}}\left(A_{k}-z_{k}\right) \xi_{F \backslash\{k\}}=0 .
$$


In particular, for $F=\{j\}$ we have

$$
\left(A_{j}-z_{j}\right) P W I s=\left(A_{j}-z_{j}\right) \xi_{\emptyset}=-\bar{\partial} \xi_{\{j\}} .
$$

Thus

$$
\begin{aligned}
& \int_{\mathbb{C}^{n}} \bar{\partial} \varphi f \cdot\left(A_{j}-z_{j}\right) P W I s \wedge \mathrm{d} z=-\int_{\mathbb{C}^{n}} \bar{\partial} \varphi f \bar{\partial} \xi_{\{j\}} \wedge \mathrm{d} z \\
& =-\int_{\mathbb{C}^{n}} \bar{\partial}\left(\varphi \bar{\partial} f \xi_{\{j\}}-\bar{\partial} \varphi f \xi_{\{j\}}\right) \wedge \mathrm{d} z=0
\end{aligned}
$$

by the Stokes theorem. Hence $g(A)=A_{j} f(A)$.

Q.E.D.

Proposition 13. Let $A=\left(A_{1}, \ldots, A_{n}\right) \in \mathcal{B}(X)^{n}, B=\left(B_{1}, \ldots, B_{m}\right) \in \mathcal{B}(X)^{m}$. Suppose that $(A, B)=\left(A_{1}, \ldots, A_{n}, B_{1}, \ldots, B_{m}\right)$ is a commuting $(n+m)$-tuple and let $f$ and $g$ be functions analytic on a neighbourhood of $\sigma_{T}(A)$ and $\sigma_{T}(B)$, respectively. Define function $h$ by $h(z, w)=f(z) \cdot g(w)$. Then $h(A, B)=g(B) f(A)$.

Proof. Write $z=\left(z_{1}, \ldots, z_{n}\right)$ and $w=\left(w_{1}, \ldots, w_{m}\right)$. Denote by $\bar{\partial}_{z}, \bar{\partial}_{w}$ and $\bar{\partial}_{z, w}$ the $\bar{\partial}$ operator corresponding to $z, w$ and $(z, w)$, respectively. We associate with $B$ another system $t=\left(t_{1}, \ldots, t_{m}\right)$ of exterior indeterminates when defining the operator $\delta_{B-w}$.

Choose mappings $W_{A}, W_{B}$ and $W_{A, B}$ corresponding to the tuples $A, B$ and $(A, B)$. Let $\Delta^{\prime}$ and $\Delta^{\prime \prime}$ be compact neighbourhoods of $\sigma_{T}(A)$ and $\sigma_{T}(B)$ contained in the domains of definition of $f$ and $g$, respectively. Let $\varphi, \psi$ and $\chi$ be $C^{\infty}$-functions equal to 0 on a neighbourhood of $\sigma_{T}(A)\left(\sigma_{T}(B)\right.$ and $\left.\sigma_{T}(A, B)\right)$, and to 1 on $\mathbb{C}^{n} \backslash \Delta^{\prime}\left(\mathbb{C}^{m} \backslash \Delta^{\prime \prime}\right.$ and $\mathbb{C}^{n+m} \backslash \Delta^{\prime} \times \Delta^{\prime \prime}$, respectively).

Denote by $P_{s}$ and $P_{t}$ the projections which annihilate all terms containing at least one of variables $s_{1}, \ldots, s_{n}\left(t_{1}, \ldots, t_{m}\right.$, respectively) and leave invariant the remaining terms. Set $P=P_{s} P_{t}$.

Let $x \in X$. We have

$$
f(A) x=\frac{-1}{(2 \pi i)^{n}} \int_{\mathbb{C}^{n}} \bar{\partial}_{z} \varphi P_{s} W_{A} f x s \wedge \mathrm{d} z=\frac{-1}{(2 \pi i)^{n}} \int_{\mathbb{C}^{n}} P_{s} \xi \wedge \mathrm{d} z
$$

where $\xi=\left(\bar{\partial}_{z}+\delta_{A-z}\right) \varphi W_{A} f x s-f x s$. On $\mathbb{C}^{n} \backslash \Delta^{\prime}$ we have $\varphi \equiv 1$ and so $\xi \equiv 0$. Thus $\operatorname{supp} \xi$ is compact. Further

$$
g(B) f(A) x=\frac{1}{(2 \pi i)^{n+m}} \int_{\mathbb{C}^{m}} P_{t}\left(\bar{\partial}_{w}+\delta_{B-w}\right) \psi W_{B} g\left(\int_{\mathbb{C}^{n}} P_{s} \xi \wedge \mathrm{d} z\right) t \wedge \mathrm{d} w .
$$

Since $W_{B}$ is not linear, we cannot interchange it with the inner integral. However, consider the form

$$
\eta=\left(\bar{\partial}_{w}+\delta_{B-w}\right) \psi W_{B} g\left(\int_{\mathbb{C}^{n}} P_{s} \xi \wedge \mathrm{d} z\right) t-\left(\bar{\partial}_{w}+\delta_{B-w}\right) \psi \int_{\mathbb{C}^{n}} W_{B}\left(P_{s} g \xi \wedge \mathrm{d} z \wedge t\right)
$$

where $W_{B}$ is extended to $\Lambda\left[\mathrm{d} \bar{z}, \mathrm{~d} z, t, \mathrm{~d} \bar{w}, C^{\infty}\left(\mathbb{C}^{n} \times\left(\mathbb{C}^{m} \backslash \sigma_{T}(B)\right), X\right)\right]$ in the obvious way. Clearly $\left(\bar{\partial}_{w}+\delta_{B-w}\right) \eta=0$ and $\operatorname{supp} \eta$ is disjoint with $\sigma_{T}(B)$. On $\mathbb{C}^{m} \backslash \Delta^{\prime \prime}$ we have $\psi \equiv 1$ and $\eta \equiv 0$ since $\operatorname{supp} W_{B}\left(P_{s} g \xi \wedge \mathrm{d} z \wedge t\right) \subset \operatorname{supp} \xi \times \mathbb{C}^{m}$ and we can interchange $\bar{\partial}_{w}$ with the second integral. Thus $\int_{\mathbb{C}^{m}} P_{t} \eta \wedge \mathrm{d} w=0$ and we have

$$
(2 \pi i)^{n+m} g(B) f(A) x=\int_{\mathbb{C}^{m}} P_{t}\left(\bar{\partial}_{w}+\delta_{B-w}\right) \psi \int_{\mathbb{C}^{n}} W_{B}\left(P_{s} g \xi \wedge \mathrm{d} z \wedge t\right) \wedge \mathrm{d} w .
$$


On the other hand, $-(2 \pi i)^{m+n} h(A, B) x=\int P \eta_{1} \wedge \mathrm{d} z \wedge \mathrm{d} w$ where

$$
\eta_{1}=\left(\bar{\partial}_{z, w}+\delta_{A-z, B-w}\right) \chi W_{A, B} h x s \wedge t-h x s \wedge t
$$

Clearly $\operatorname{supp} \eta_{1}$ is compact.

Set

$$
\eta_{2}=\left(\bar{\partial}_{z, w}+\delta_{A-z, B-w}\right) \psi W_{A, B} g \xi \wedge t-g \xi \wedge t .
$$

Clearly supp $\eta_{2} \subset \operatorname{supp} \xi \times \mathbb{C}^{m}$. Moreover, if $\psi \equiv 1$ then $\eta_{2} \equiv 0$, so $\operatorname{supp} \eta_{2}$ is compact. On a neighbourhood of $\sigma_{T}(A, B)$ we have $\eta_{2}=-g \xi \wedge t=f g x s \wedge t=-\eta_{1}$. By Proposition 9, we have $\int P\left(\eta_{1}+\eta_{2}\right) \wedge \mathrm{d} z \wedge \mathrm{d} w=0$ and so

$$
\begin{aligned}
& (2 \pi i)^{m+n} h(A, B) x=\int_{\mathbb{C}^{n+m}} P \eta_{2} \wedge \mathrm{d} z \wedge \mathrm{d} w \\
& =(-1)^{m n} \int_{\mathbb{C}^{m}}\left(\int_{\mathbb{C}^{n}} P_{t}\left(\bar{\partial}_{z, w}+\delta_{B-w}\right) \psi P_{s} W_{A, B} g \xi \wedge t \wedge \mathrm{d} z\right) \wedge \mathrm{d} w
\end{aligned}
$$

by the Fubini theorem (the factor $(-1)^{m n}$ is caused by convention (4) defining the Lebesgue measures in $\mathbb{C}^{n}, \mathbb{C}^{m}$ and $\mathbb{C}^{m+n}$, respectively). By the Stokes theorem we have

$$
(2 \pi i)^{m+n} h(A, B) x=\int_{\mathbb{C}^{m}} P_{t}\left(\bar{\partial}_{w}+\delta_{B-w}\right) \psi\left(\int_{\mathbb{C}^{n}} P_{s} W_{A, B} g \xi \wedge \mathrm{d} z \wedge t\right) \wedge \mathrm{d} w .
$$

Consider the form

$$
\eta_{3}=\left(\bar{\partial}_{w}+\delta_{B-w}\right) \psi \int_{\mathbb{C}^{n}} P_{s} W_{A, B} g \xi \wedge \mathrm{d} z \wedge t-\left(\bar{\partial}_{w}+\delta_{B-w}\right) \psi \int_{\mathbb{C}^{n}} W_{B}\left(P_{s} g \xi \wedge \mathrm{d} z \wedge t\right) .
$$

Clearly $\operatorname{supp} \eta_{3} \cap \sigma_{T}(B)=\emptyset$ and $\left(\bar{\partial}_{w}+\delta_{B-w}\right) \eta_{3}=0$. If $\psi \equiv 1$ then, by the Stokes theorem,

$$
\begin{array}{r}
\eta_{3}=\int_{\mathbb{C}^{n}} P_{s}\left(\bar{\partial}_{z, w}+\delta_{A-z, B-w}\right) W_{A, B} g \xi \wedge \mathrm{d} z \wedge t-\int_{\mathbb{C}^{n}} \bar{\partial}_{z} P_{s} W_{A, B} g \xi \wedge \mathrm{d} z \wedge t \\
-\int_{\mathbb{C}^{n}} P_{s} g \xi \wedge \mathrm{d} z \wedge t=\int_{\mathbb{C}^{n}} P_{s} g \xi \wedge \mathrm{d} z \wedge t-\int_{\mathbb{C}^{n}} P_{s} g \xi \wedge \mathrm{d} z \wedge t=0 .
\end{array}
$$

Thus $\int P_{t} \eta_{3} \wedge \mathrm{d} w=0$ and

$$
\begin{aligned}
(2 \pi i)^{n+m} h(A, B) x & =\int_{\mathbb{C}^{m}} P_{t}\left(\bar{\partial}_{w}+\delta_{B-w}\right) \psi \int_{\mathbb{C}^{n}} W_{B}\left(P_{s} g \xi \mathrm{d} z \wedge t\right) \wedge \mathrm{d} w \\
& =(2 \pi i)^{m+n} g(B) f(A) x
\end{aligned}
$$

by (8). Hence $h(A, B)=g(B) f(A)$.

Q.E.D.

We shall use the following simple lemma: 
Lemma 14. Let $K$ be a compact subset of $\mathbb{C}^{n}$ and let $f$ be a function analytic on an open neighbourhood of $K$. Then there are functions $h_{j}(j=1, \ldots, n)$ analytic on a neighbourhood of the set $D=\{(z, z): z \in K\}$ such that

$$
f(z)-f(w)=\sum_{j=1}^{n}\left(z_{j}-w_{j}\right) \cdot h_{j}(z, w) .
$$

Proof. For $j=1, \ldots, n$ define $g_{j}$ by

$$
g_{j}\left(z_{1}, \ldots, z_{n}, w_{1}, \ldots, w_{n}\right)=f\left(z_{1}, \ldots, z_{j}, w_{j+1}, \ldots, w_{n}\right)-f\left(z_{1}, \ldots, z_{j-1}, w_{j}, \ldots, w_{n}\right) .
$$

It is easy to see that $g_{j}$ is defined and analytic on a neighbourhood of $D$.

Let $h_{j}(z, w)=\frac{g_{j}(z, w)}{z_{j}-w_{j}}$. Clearly $h_{j}$ is analytic at each point $(z, w)$ with $z_{j} \neq w_{j}$. By the Weierstrass division theorem (see [7], p. 70), $h_{j}$ can be defined and is analytic also on a neighbourhood of each point $(z, w)$ with $z_{j}=w_{j}$. Thus $h_{j}$ is analytic on a neighbourhood of $D$. Clearly

$$
\sum_{j=1}^{n}\left(z_{j}-w_{j}\right) \cdot h_{j}(z, w)=\sum_{j=1}^{n} g_{j}(z, w)=f(z)-f(w) .
$$

Q.E.D.

Denote by $\mathcal{A}_{K}$ the algebra of all functions analytic on a neighbourhood of a compact set $K \subset \mathbb{C}^{n}$ (more precisely, the algebra of all germs of functions analytic on a neighbourhood of $K)$.

Theorem 15. Let $A=\left(A_{1}, \ldots, A_{n}\right)$ be an $n$-tuple of mutually commuting operators on $X$. Then:

(i) the mapping $f \mapsto f(A)$ is linear and multiplicative, i.e., the Taylor functional calculus is a homomorphism from $\mathcal{A}_{\sigma_{T}(A)}$ to $\mathcal{B}(X)$;

(ii) if $p$ is a polynomial, $p(z)=\sum_{\alpha \in \mathbb{Z}_{+}^{n}} c_{\alpha} z^{\alpha}$ then $p(A)=\sum_{\alpha \in \mathbb{Z}_{+}^{n}} c_{\alpha} A^{\alpha}$;

(iii) if $f_{n} \rightarrow f$ uniformly on a compact neighbourhood of $\sigma_{T}(A)$ then $f_{n}(A) \rightarrow f(A)$ in the norm topology;

(iv) $f(A) \in(A)^{\prime \prime}$ for each $f \in A_{\sigma_{T}(A)}$.

Proof. (i) The linearity of the mapping $f \mapsto f(A)$ is clear. Let $f$ and $g$ be functions analytic on a neighbourhood of $\sigma_{T}(A)$. Consider the $(2 n)$-tuple $(A, A)$. It is easy to see that $\sigma_{T}(A, A)=\left\{(z, z): z \in \sigma_{T}(A)\right\}$. Define functions $h_{1}(z, w)=f(z) g(w)$ and $h_{2}(z, w)=f(z) g(z)$. By Lemma 14, we can write $g(z)-g(w)=\sum_{i=1}^{n}\left(z_{i}-w_{i}\right) q_{i}(z, w)$ for some functions $q_{1}, \ldots, q_{n}$ analytic on a neighbourhood of $\sigma_{T}(A, A)$. By Proposition 13, we have $h_{1}(A, A)=f(A) g(A)$ and $h_{2}(A, A)=(f g)(A)$. Thus, by Proposition 12 ,

$$
(f g)(A)-f(A) g(A)=h_{2}(A, A)-h_{1}(A, A)=\sum_{i=1}^{n}\left(A_{i}-A_{i}\right)\left(f q_{i}\right)(A, A)=0 .
$$

Hence $(f g)(A)=f(A) g(A)$. 
(ii) The statement follows from Propositions 11 and 13.

(iii) follows from the definition.

(iv) Let $S \in \mathcal{B}(X)$ be an operator commuting with $A_{1}, \ldots, A_{n}$. By Proposition 13 , it is possible to consider $f(A)$ to be a function of the $(n+1)$-tuple $\left(A_{1}, \ldots, A_{n}, S\right)$. Therefore $f(A)$ commutes with its argument $S$. Hence $f(A) \in(A)^{\prime \prime}$.

Q.E.D.

It follows from the general theory [23] that the Taylor spectrum satisfies the spectral mapping property for all polynomials (and consequently, for all functions that can be approximated by polynomials uniformly on a neighbourhood of the Taylor spectrum). In fact the spectral mapping property is true for all analytic functions. To show this, we need the following lemma:

Lemma 16. Let $A=\left(A_{1}, \ldots, A_{n}\right)$ be a commuting $n$-tuple of operators on $X$, let $c=\left(c_{1}, \ldots, c_{n}\right) \in \sigma_{T}(A)$ and let $f$ be a function analytic on a neighbourhood of $\sigma_{T}(A)$. Consider exterior indeterminates $t=\left(t_{1}, \ldots, t_{n}\right)$ and operator $\delta_{A-c, t}: \Lambda[t, X] \rightarrow \Lambda[t, X]$ defined by $\delta_{A-c, t} \psi=\sum_{j=1}^{n}\left(A_{j}-c_{j}\right) t_{j} \wedge \psi(\psi \in \Lambda[t, X])$. Let $\eta_{0} \in \operatorname{Ker} \delta_{A-c, t}$. Then $(f(A)-f(c)) \eta_{0} \in \delta_{A-c, t} \Lambda[t, X]$.

Proof. Without loss of generality we can assume that $\eta_{0}$ is homogeneous of degree $p$, $0 \leq p \leq n$.

To define $f(A)$, consider exterior indeterminates $s=\left(s_{1}, \ldots, s_{n}\right)$, the mapping $\delta_{A-z}$ acting on $\Lambda\left[s, \mathrm{~d} \bar{z}, C^{\infty}\left(\mathbb{C}^{n} \backslash \sigma_{T}(A), X\right)\right]$ defined by $\delta_{A-z} \psi=\sum_{j=1}^{n}\left(A_{j}-z_{j}\right) s_{j} \wedge \psi$ and the mapping $W_{A}$ corresponding to $A$. We can lift $\delta_{A-z}$ and $W_{A}$ to the space $\Lambda\left[s, t, \mathrm{~d} \bar{z}, C^{\infty}\left(\mathbb{C}^{n} \backslash \sigma_{T}(A), X\right)\right]$ in the natural way. Note that $\delta_{A-z}$ and $W_{A}$ are connected with variables $s$; the mapping $\delta_{A-c, t}$ is related to variables $t$.

Set $\eta=f \eta_{0} \wedge s$ and $\xi_{1}=\sum_{k=0}^{n}(-1)^{k} W_{A}\left(\delta_{A-c, t} W_{A}\right)^{k} \eta$. We show by induction that $\left(\bar{\partial}+\delta_{A-z}\right)\left(\delta_{A-c, t} W_{A}\right)^{k} \eta=0$ for all $k$. This is clear for $k=0$; for $k \geq 1$ we have

Hence

$$
\begin{aligned}
\left(\bar{\partial}+\delta_{A-z}\right)\left(\delta_{A-c, t} W_{A}\right)^{k} \eta & =-\delta_{A-c, t}\left(\bar{\partial}+\delta_{A-z}\right) W_{A}\left(\delta_{A-c, t} W_{A}\right)^{k-1} \eta \\
& =-\delta_{A-c, t}\left(\delta_{A-c, t} W_{A}\right)^{k-1} \eta=0 .
\end{aligned}
$$

$$
\begin{aligned}
& \left(\bar{\partial}+\delta_{A-z}+\delta_{A-c, t}\right) \xi_{1}=\left(\bar{\partial}+\delta_{A-z}\right) \xi_{1}+\delta_{A-c, t} \xi_{1} \\
& =\sum_{k=0}^{n}(-1)^{k}\left(\delta_{A-c, t} W_{A}\right)^{k} \eta+\sum_{k=0}^{n}(-1)^{k}\left(\delta_{A-c, t} W_{A}\right)^{k+1} \eta=\eta
\end{aligned}
$$

since $\left(\delta_{A-c, t} W_{A}\right)^{n+1}=0$. Let $\varphi$ be a $C^{\infty}$-function equal to 0 on a neighbourhood of $\sigma_{T}(A)$ such that $\operatorname{supp}(1-\varphi)$ is compact. Let $P_{s}$ be the projection annihilating all terms that contain at least one of the variables $s_{1}, \ldots, s_{n}$ and leaving invariant all other terms.

Consider the integral

$$
\int\left(\bar{\partial}+\delta_{A-c, t}\right) P_{s} \varphi \xi_{1} \wedge \mathrm{d} z=\int\left(\bar{\partial}+\delta_{A-c, t}\right) P_{s} \varphi\left(W_{A} \eta-W_{A} \delta_{A-c, t} W_{A} \eta+\cdots\right) \wedge \mathrm{d} z .
$$

Since $W_{A}\left(\delta_{A-c, t} W_{A}\right)^{k} \eta$ has degree $p+k$ in $t$ and $n-k-1$ in $(s, \mathrm{~d} \bar{z})$, the only relevant term in the integral above is $W_{A} \eta$. Thus

$$
\begin{aligned}
& \int\left(\bar{\partial}+\delta_{A-c, t}\right) P_{s} \varphi \xi_{1} \wedge \mathrm{d} z=\int\left(\bar{\partial}+\delta_{A-c, t}\right) P_{s} \varphi W_{A} \eta \wedge \mathrm{d} z \\
& =\int \bar{\partial} P_{s} \varphi W_{A} \eta \wedge \mathrm{d} z=-(2 \pi i)^{n} f(A) \eta_{0} .
\end{aligned}
$$


Consider now the $n$-tuple $B=\left(c_{1} I, \ldots, c_{n} I\right) \in \mathcal{B}(X)^{n}$. Since $f$ can be approximated by polynomials uniformly on a neighbourhood of $c$, we note that $f(B)=f(c) \cdot I$.

As above, consider mappings $\delta_{B-z}$ and $W_{B}$ connected with variables $s$. $\eta$ and

Let $\xi_{2}=\sum_{k=0}^{n}(-1)^{k} W_{B}\left(\delta_{A-c, t} W_{B}\right)^{k} \eta$. As above, we have $\left(\bar{\partial}+\delta_{B-z}+\delta_{A-c, t}\right) \xi_{2}=$

$$
\begin{aligned}
& \int\left(\bar{\partial}+\delta_{A-c, t}\right) P_{s} \varphi \xi_{2} \wedge \mathrm{d} z=\int\left(\bar{\partial}+\delta_{A-c, t}\right) P_{s} \varphi W_{B} \eta \wedge \mathrm{d} z \\
& =\int \bar{\partial} P_{s} \varphi W_{B} \eta \wedge \mathrm{d} z=-(2 \pi i)^{n} f(B) \eta_{0}=-(2 \pi i)^{n} f(c) \eta_{0} .
\end{aligned}
$$

To show that $(f(A)-f(c)) \eta_{0} \in \delta_{A-c, t} \Lambda[t, X]$, consider the linear mapping $U$ acting on $\Lambda\left[s, t, \mathrm{~d} \bar{z}, C^{\infty}\left(\mathbb{C}^{n} \backslash \sigma_{T}(A), X\right)\right]$ defined by

$$
U\left(t_{i_{1}} \wedge \cdots \wedge t_{i_{m}} \wedge \psi\right)=\left(t_{i_{1}}-s_{i_{1}}\right) \wedge \cdots \wedge\left(t_{i_{m}}-s_{i_{m}}\right) \wedge \psi
$$

for all $i_{1}, \ldots, i_{m}$ and $\psi \in \Lambda\left[s, \mathrm{~d} \bar{z}, C^{\infty}\left(\mathbb{C}^{n} \backslash \sigma_{T}(A), X\right)\right]$. Clearly $P_{s} U=P_{s}$ and, for each $\psi \in \Lambda\left[s, t, \mathrm{~d} \bar{z}, C^{\infty}\left(\mathbb{C}^{n} \backslash \sigma_{T}(A), X\right)\right]$,

$$
\begin{aligned}
& U\left(\bar{\partial}+\delta_{A-z}+\delta_{A-c, t}\right) \psi \\
& =\bar{\partial} U \psi+\sum\left(A_{j}-z_{j}\right) s_{j} \wedge U \psi+\sum\left(A_{j}-c_{j}\right)\left(t_{j}-s_{j}\right) \wedge U \psi \\
& =\left(\bar{\partial}+\delta_{B-z}+\delta_{A-c, t}\right) U \psi
\end{aligned}
$$

We have

$$
\begin{aligned}
& -(2 \pi i)^{n} f(A) \eta_{0}=\int\left(\bar{\partial}+\delta_{A-c, t}\right) P_{s} \varphi \xi_{1} \wedge \mathrm{d} z=\int P_{s}\left(\bar{\partial}+\delta_{A-z}+\delta_{A-c, t}\right) \varphi \xi_{1} \wedge \mathrm{d} z \\
& =\int P_{s} U\left(\bar{\partial}+\delta_{A-z}+\delta_{A-c, t}\right) \varphi \xi_{1} \wedge \mathrm{d} z=\int P_{s}\left(\bar{\partial}+\delta_{B-z}+\delta_{A-c, t}\right) \varphi U \xi_{1} \wedge \mathrm{d} z .
\end{aligned}
$$

Thus

$$
-(2 \pi i)^{n}(f(A)-f(c)) \eta_{0}=\int P_{s}\left(\bar{\partial}+\delta_{B-z}+\delta_{A-c, t}\right) \varphi\left(U \xi_{1}-\xi_{2}\right) \wedge \mathrm{d} z=\int P_{s} \theta \wedge \mathrm{d} z
$$

where $\theta=\left(\bar{\partial}+\delta_{B-z}+\delta_{A-c, t}\right) \varphi\left(U \xi_{1}-\xi_{2}\right)$. If $\varphi \equiv 1$ then $\theta=\left(\bar{\partial}+\delta_{B-z}+\delta_{A-c, t}\right) U \xi_{1}-\eta=$ $U\left(\bar{\partial}+\delta_{A-z}+\delta_{A-c, t}\right) \xi_{1}-\eta=U \eta-\eta=0$; so $\operatorname{supp} \theta$ is compact. Furthermore, $\theta$ can be written as $\theta=\left(\bar{\partial}+\delta_{B-z}+\delta_{A-c, t}\right) \psi$ for some form $\psi \in \Lambda\left[s, t, \mathrm{~d} \bar{z}, C^{\infty}\left(\mathbb{C}^{n}, X\right)\right]$ with compact support. Indeed, by Remark 8 , there exists a form $\vartheta \in \Lambda\left[s, t, \mathrm{~d} \bar{z}, \mathrm{~d} \bar{w}, C^{\infty}\left(\mathbb{C}^{2 n}, X\right)\right]$ with $\operatorname{supp} \vartheta \subset \operatorname{supp} \theta \times \mathbb{C}^{n} \operatorname{such}$ that $\left(\bar{\partial}_{z, w}+\delta_{B-z}+\delta_{A-c, t}\right) \vartheta=\theta$.

Set $\psi(z)=\vartheta_{0}(z, c)$ where $\vartheta_{0}$ is the part of $\vartheta$ containing none of the variables $\mathrm{d} \bar{w}_{j}$. Then $\operatorname{supp} \psi \subset \operatorname{supp} \theta$ and $\left(\bar{\partial}_{z}+\delta_{B-z}+\delta_{A-c, t}\right) \psi=\theta$. By the Stokes theorem,

$$
\begin{aligned}
& \int P_{s} \theta \wedge \mathrm{d} z=\int P_{s}\left(\bar{\partial}_{z}+\delta_{B-z}+\delta_{A-c, t}\right) \psi \wedge s \wedge \mathrm{d} z \\
& =\int \bar{\partial}_{z} P_{s} \psi \wedge d c+\int P_{s} \delta_{A-c, t} \psi \wedge \mathrm{d} z=\delta_{A-c, t} \int P_{s} \psi \wedge \mathrm{d} z \in \delta_{A-c, t} \Lambda[t, X] .
\end{aligned}
$$

Q.E.D. 
Proposition 17. Let $A=\left(A_{1}, \ldots, A_{n}\right)$ be a commuting $n$-tuple of operators on $X$, $c=\left(c_{1}, \ldots, c_{n}\right) \in \sigma_{T}(A)$ and let $f$ be a function analytic on a neighbourhood of $\sigma_{T}(A)$. Then the $(n+1)$-tuple $\left(A_{1}-c_{1}, \ldots, A_{n}-c_{n}, f(A)\right)$ is Taylor regular if and only if $f(c) \neq 0$.

Proof. To the $(n+1)$-tuple $(A-c, f(A))$ we relate exterior variables $s_{1}, \ldots, s_{n+1}$. Write for short $s=\left(s_{1}, \ldots, s_{n}\right)$. Let $\delta_{A-c}: \Lambda[s, X] \rightarrow \Lambda[s, X]$ be be defined by $\delta_{A-c} \psi=$ $\sum\left(A_{j}-c_{j}\right) s_{j} \wedge \psi \quad(\psi \in \Lambda[s, X])$. Clearly $\Lambda\left[s, s_{n+1}, X\right]=\Lambda[s, X] \oplus s_{n+1} \wedge \Lambda[s, X]$. The operator $\delta_{A-c, f(A)}$ corresponding to the $(n+1)$-tuple $(A-c, f(A))$ can be written in this decomposition in the matrix form

$$
\delta_{A-c, f(A)}=\left(\begin{array}{cc}
\delta_{A-c} & 0 \\
f(A) & -\delta_{A-c}
\end{array}\right) .
$$

We distinguish two cases:

(a) $f(c)=0$.

Since $c \in \sigma_{T}(A)$, there is a $\psi \in \Lambda[s, X]$ such that $\delta_{A-c} \psi=0$ and $\psi \notin \delta_{A-c} \Lambda[s, X]$. By the preceding lemma, there is an $\eta \in \Lambda[s, X]$ such that $f(A) \psi=\delta_{A-c} \eta$. Then $\delta_{A-c, f(A)}\left(\psi+s_{n+1} \wedge \eta\right)=0$ and $\left(\psi+s_{n+1} \wedge \eta\right) \notin \delta_{A-c, f(A)} \Lambda\left[s, s_{n+1}, X\right]$ since $\psi \notin$ $\delta_{A-c} \Lambda[s, X]$. Thus the $(n+1)$-tuple $(A-c, f(A))$ is Taylor singular.

(b) $f(c) \neq 0$. Without loss of generality we can assume that $f(c)=1$.

Let $\psi, \xi \in \Lambda[s, X], \delta_{A-c, f(A)}\left(\psi+s_{n+1} \wedge \xi\right)=0$. Then $\delta_{A-c} \psi=0$ and $f(A) \psi-$ $\delta_{A-c} \xi=0$. By the preceding lemma, $f(A) \psi-\psi \in \delta_{A-c} \Lambda[s, X]$. Since $f(A) \psi \in$ $\delta_{A-c} \Lambda[s, X]$, we have $\psi=\delta_{A-c} \eta$ for some $\eta \in \Lambda[s, X]$.

Further $\delta_{A-c}(f(A) \eta-\xi)=f(A) \psi-\delta_{A-c} \xi=0$. Thus there is an $\theta \in \Lambda[s, X]$ with $f(A)(f(A) \eta-\xi)-(f(A) \eta-\xi)=\delta_{A-c} \theta$. Set $\eta^{\prime}=\eta-(f(A) \eta-\xi)$. Then $\delta_{A-c} \eta^{\prime}=\delta_{A-c} \eta=$ $\psi$ and $f(A) \eta^{\prime}-\delta_{A-c} \theta=f(A) \eta-f(A)(f(A) \eta-\xi)+\delta_{A-c} \theta=f(A) \eta-(f(A) \eta-\xi)=\xi$. Hence $\delta_{A-c, f(A)}\left(\eta^{\prime}-s_{n+1} \wedge \theta\right)=\left(\psi+s_{n+1} \wedge \xi\right)$ and the $(n+1)$-tuple $(A-c, f(A))$ is Taylor regular.

Q.E.D.

Theorem 18. (spectral mapping property) Let $A=\left(A_{1}, \ldots, A_{n}\right)$ be a commuting $n$-tuple of operators on $X$ and let $f=\left(f_{1}, \ldots, f_{m}\right)$ be an $m$-tuple of functions analytic on a neighbourhood of $\sigma_{T}(A)$. Then $\sigma_{T}(f(A))=f \sigma_{T}(A)$.

Proof. Consider the commutative Banach algebra $\mathcal{A}$ generated by $A_{1}, \ldots, A_{n}, I$ and $f_{1}(A), \ldots, f_{m}(A)$. Since the restriction of $\sigma_{T}$ to $\mathcal{A}$ satisfies the projection property, by [23] there is a compact subset $K$ of the maximal ideal space of $\mathcal{A}$ such that $\sigma_{T}(B)=$ $\{\varphi(B): \varphi \in K\}$ for each tuple $B=\left(B_{1}, \ldots, B_{k}\right) \subset \mathcal{A}$.

Fix $\varphi \in K$ and $i, 1 \leq i \leq m$. Let $c_{j}=\varphi\left(A_{j}\right) \quad(j=1, \ldots, n)$ and $c=\left(c_{1}, \ldots, c_{n}\right) \in$ $\sigma_{T}(A)$. Then the $(n+1)$-tuple $\left(A_{1}-c_{1}, \ldots, A_{n}-c_{n}, f_{i}(A)-\varphi\left(f_{i}(A)\right)\right)$ is Taylor singular. By Proposition 17, $f_{i}(c)-\varphi\left(f_{i}(A)\right)=0$, i.e., $\varphi\left(f_{i}(A)\right)=f_{i}(\varphi(A))$. Then

$$
\begin{aligned}
& \sigma_{T}(f(A))=\left\{\left(\varphi\left(f_{1}(A), \ldots \varphi\left(f_{m}(A)\right)\right): \varphi \in K\right\}=\left\{\left(f_{1}(\varphi(A)), \ldots, f_{m}(\varphi(A))\right): \varphi \in K\right\}\right. \\
& =\left\{f(c): c \in \sigma_{T}(A)\right\}=f \sigma_{T}(A) .
\end{aligned}
$$

Q.E.D.

Theorem 19. (superposition property [13], [6]) Let $A=\left(A_{1}, \ldots, A_{m}\right)$ be a commuting $n$-tuple of operators on $X$, let $f=\left(f_{1}, \ldots, f_{m}\right)$ be an $m$-tuple of function analytic on a 
neighbourhood of $\sigma_{T}(A)$, let $B=f(A)$, let $g$ be a function analytic on a neighbourhood of $\sigma_{T}(B)$ and let $h(z)=g\left(f_{1}(z), \ldots, f_{m}(z)\right)$. Then $h(A)=g(B)$.

Proof. By Lemma 14, $g(v)-g(w)=\sum_{j=1}^{m}\left(v_{j}-w_{j}\right) r_{j}(v, w)$ for some functions $r_{1}, \ldots, r_{m}$ analytic on a neighbourhood of the set $\left\{(v, v): v \in \sigma_{T}(B)\right\}$. Thus

$$
g(f(z))-g(w)=\sum_{j=1}^{m}\left(f_{j}(z)-w_{j}\right) r_{j}^{\prime}(z, w)
$$

where $\left.r_{j}^{\prime}(z, w)=r_{j}(f(z), w)\right)$ and functions $r_{j}^{\prime}$ are analytic on a neighbourhood of the set $\left\{(z, f(z)): z \in \sigma_{T}(A)\right\}=\sigma_{T}(A, f(A))$. Thus $h(A)-g(B)=\sum_{j=1}^{m}\left(f_{j}(A)-\right.$ $\left.B_{j}\right) r_{j}^{\prime}(A, B)=0$. Hence $h(A)=g(B)$.

Q.E.D.

\section{Concluding Remarks}

1. There are many variants of formulas (5), (6) defining the Taylor functional calculus that differ from each other in the sign in front of the integral. There are several sources of differences:

(a) Instead of the $n$-tuple $A-z=\left(A_{1}-z_{1}, \ldots, A_{n}-z_{n}\right)$ it is possible to consider the $n$-tuple $z-A$ (which appears naturally in the Cauchy formula). In this approach an additional factor $(-1)^{n}$ in front of the integral (5) would appear.

(b) Instead of (4) it is possible to use convention that the Lebesgue measure in $\mathbb{C}^{n}$ is $(2 i)^{-n} \mathrm{~d} \bar{z}_{1} \wedge \mathrm{d} z_{1} \wedge \cdots \wedge \mathrm{d} \bar{z}_{n} \wedge \mathrm{d} z_{n}$. With this convention the Fubini theorem becomes more natural. In formula (5), however, an additional factor $(-1)\left(\begin{array}{l}n \\ 2\end{array}\right)$ would appear.

(c) It is also possible to modify the definition of the mappings $\delta_{A}^{p}$ in the Koszul complex as in [10]: $\delta_{A}^{p} x s_{i_{1}} \wedge \cdots \wedge s_{i_{p}}=\sum_{j} A_{j} x s_{i_{1}} \wedge \cdots \wedge s_{i_{p}} \wedge s_{j}$. This convention results also in an additional factor $(-1)^{\left(\begin{array}{c}n \\ 2\end{array}\right)}$ in formula (5).

2. For Hilbert space operators it is possible to choose $V=\left(\delta_{A-z}+\delta_{A-z}^{*}\right)^{-1}$, see [20], [21], [22]. Formula (7) is then quite explicit.

3. The split-spectrum $\sigma_{S}(A)$ of the $n$-tuple $A=\left(A_{1}, \ldots, A_{n}\right) \in \mathcal{B}(X)^{n}$ is defined as the set of all $\lambda \in \mathbb{C}^{n}$ such that either $\operatorname{Im} \delta_{A-\lambda} \neq \operatorname{Ker} \delta_{A-\lambda}$ or $\operatorname{Im} \delta_{A-\lambda}$ is not complemented in $\Lambda[s, X]$. In general $\sigma_{S}(A)$ is bigger than $\sigma_{T}(A)$, see [12] (in Hilbert spaces these two spectra coincide).

On the complement of $\sigma_{S}(A)$ it is possible to find bounded linear generalized inverses $V(z)$, see [9]. Thus for functions analytic on a neighbourhood of the splitspectrum the proof of basic properties of the Taylor functional calculus becomes simpler. The linearity of $f(A)$ is clear and also the proofs of multiplicativity of the functional calculus and the spectral mapping property are simpler.

4. As in Theorem 18, it is possible to prove the spectral mapping property for functions analytic on a neighbourhood of the Taylor spectrum for each spectral system which is contained in the Taylor spectrum. In particular, this applies to the spectra of Słodkowski and the essential Taylor spectrum, see [14].

5. An interesting problem is to generalize the Taylor spectrum for Banach algebras. 
Let $a=\left(a_{1}, \ldots, a_{n}\right)$ be a commuting $n$-tuple of elements of a Banach algebra. Denote by $L_{a}=\left(L_{a_{1}}, \ldots, L_{a_{n}}\right)$ the $n$-tuple of left multiplication operators acting on $\mathcal{A}$. A natural idea is to define the Taylor spectrum of $a$ as $\sigma_{T}\left(L_{a}\right)$. However, if $\mathcal{A}=\mathcal{B}(X)$ is the algebra of operators on a Banach space $X$ and $A \in \mathcal{B}(X)^{n}$ a commuting $n$-tuple, then $\sigma_{T}\left(L_{A}\right)=\sigma_{S}(A)$. Thus this simple way does not produce the Taylor spectrum in $\mathcal{B}(X)$.

In fact in this situation $A$ can be considered also as a commuting $n$-tuple of elements of $\mathcal{H}(X)$ where $\mathcal{H}(X)$ satisfies all axioms of Banach algebras except one of the distributive laws; let us call such objects semi-distributive algebras. Define $L_{A}^{\prime}=\left(L_{A_{1}}^{\prime}, \ldots, L_{A_{n}}^{\prime}\right) \in \mathcal{B}(\mathcal{H}(X))^{n}$ by $L_{A_{i}}^{\prime} \varphi=A_{i} \varphi \quad(\varphi \in \mathcal{H}(X))$; clearly $L_{A_{i}}^{\prime}$ is an extension of $L_{A_{i}}$. It is easy to check now that $\sigma_{T}\left(L_{A}^{\prime}\right)=\sigma_{T}(A)$.

It seems that the natural setting for the Taylor spectrum in algebras is to define it for commuting $n$-tuples $a=\left(a_{1}, \ldots, a_{n}\right)$ of elements of a semi-distributive algebra $\mathcal{A}$ that lie in the "distributive center" of $\mathcal{A}$ (more precisely, $a_{i}(b+c)=a_{i} b+a_{i} c$ for all $b, c \in \mathcal{A}, 1 \leq i \leq n$. For such an $n$-tuple, $L_{a_{i}}: \mathcal{A} \rightarrow \mathcal{A}$ defined by $L_{a_{i}} b=a_{i} b \quad(b \in \mathcal{A})$ is a linear operator and we can define the Taylor spectrum of $a$ as the Taylor spectrum of $L_{a}=\left(L_{a_{1}}, \ldots, L_{a_{n}}\right) \in \mathcal{B}(\mathcal{A})^{n}$.

\section{References}

1. D. W. Albrecht, Integral formulae for special cases of Taylor's functional calculus, Studia Math. 105 (1993), 51-68.

2. C. Ambrozie, F.-H. Vasilescu, Banach space complexes, Kluwer Academic Publishers, MAIA Series, vol. 334, 1995.

3. M. Andersson, Taylor's functional calculus for commuting operators with CauchyFantappie-Leray formulas, Int. Math. Res. Not. 6 (1997), 247-258; correction, ibidem 2 (1998), 123-124.

4. M. Andersson, Taylor's functional calculus and the resolvent mapping, Preprint Goteborg Univ., 2000.

5. M. Andersson, Various constructions of Taylor's functional calculus for commuting operators, Preprint Goteborg Univ., 2000.

6. M. Andersson, S. Sandberg, A constructive proof of the composition rule for Taylor's functional calculus, Studia Math. 142 (2000), 65-69.

7. R.C. Gunning, H. Rossi, Analytic functions of several complex variables, Prentice Hall, Englewood Cliffs, N.J. 1965.

8. A.Ya. Helemskii, The homology of Banach and topological algebras (translated from Russian), Mathematics and its applications (Soviet Series) 41, Kluver, Academic Publishers Group, Dordrecht 1989.

9. V. Kordula, V. Müller, Vasilescu-Martinelli formula for operators in Banach spaces, Studia Math. 113 (1995), 127-139.

10. R. Levi, Notes on the Taylor joint spectrum of commuting operators, Spectral Theory, Banach Center Publications, Vol. 8, PWN - Polish Scientific Publishers, Warsaw, 1982, 321-332.

11. F. Mantlik, Linear equations depending differentiably on a parameter, Integral Equations Operator Theory 13 (1990), 321-250.

12. V. Müller, The splitting spectrum differs from the Taylor spectrum, Studia Math. 123 (1997), 291-294. 
13. M. Putinar, The superposition property for Taylor's functional calculus, J. Operator Theory 7 (1982), 149-155.

14. M. Putinar, Functional calculus and the Gelfand transformation, Studia Math. 79 (1984), 83-86.

15. S. Sandberg, On non-holomorphic functional calculus for commuting operators, Preprint Goteborg Univ., 1999.

16. Z. Słodkowski, An infinite family of joint spectra, Studia Math. 61 (1977), 239-255.

17. Z. Słodkowski, Operators with closed ranges in spaces of analytic vector-valued functions, J. Funct. Anal. 28 (1986), 155-177.

18. J. L. Taylor, A joint spectrum for several commuting operators, J. Funct. Anal. 6 (1970), 172-191.

19. J. L. Taylor, Analytic-functional calculus for several commuting operators, Acta Math. 125 (1970), 1-38.

20. F.- H. Vasilescu, A Martinelli type formula for the analytic functional calculus, Rev. Roumaine Math. Pures Appl. 23 (1978), 1587-1605.

21. F.- H. Vasilescu, A multidimensional spectral theory in $C^{*}-$ algebras, Spectral Theory, Banach Center Publications, Vol. 8, PWN - Polish Scientific Publishers, Warsaw, 1982, 471-491.

22. F.- H. Vasilescu, Analytic Functional Calculus and Spectral Decompositions, Mathematics and its Applications (East European Series), Editura Academiei, Bucuresti and D. Reidel Publishing Company, Dordrecht, 1982.

23. W. Żelazko, An axiomatic approach to joint spectra I, Studia Math. 64 (1979), 249-261. 